\title{
Carbon nanotube substrates boost neuronal electrical signalling
}

Lovat Viviana $^{l}$, Davide Pantarotto ${ }^{1}$, Laura Lagostena ${ }^{1,2}$, Barbara Cacciari ${ }^{3}$, Micaela Grandolfo $^{4}$, Massimo Righi ${ }^{4}$, Giampiero Spalluto ${ }^{1,5}$, Maurizio Prato ${ }^{1,5}$, Laura Ballerini $^{2,4}$

${ }^{1}$ Department of Pharmaceutical Sciences, University of Trieste, Piazzale Europa 1, 34127, Trieste, Italy

${ }^{2}$ Physiology and Pathology Dept., Center for Neuroscience B.R.A.I.N., University of Trieste, via Fleming 22, 34127, Trieste, Italy

${ }^{3}$ Department of Pharmaceutical Sciences, University of Ferrara, via Fossato di Mortara 17-19, Ferrara, Italy

${ }^{4}$ Neurobiology Sector and Istituto Nazionale di Fisica della Materia Unit, International School for Advanced Studies (SISSA-ISAS), via Beirut 2-4, 34014 Trieste, Italy

${ }^{5}$ INSTM, Unit of Trieste, Department of Pharmaceutical Sciences, University of Trieste, Piazzale Europa 1, 34127, Trieste, Italy 


\section{Experimental}

\section{Purification of AP-MWNTs and deposition onto glass coverslips}

AP-MWNTs (100 mg), Nanostructured \& Amorphous Material Inc., USA, were suspended in $100 \mathrm{~mL}$ of DMF. The heterogeneous mixture was sonicated for $2 \mathrm{~min}$. Sarcosine $(300 \mathrm{mg})$ and heptaldehyde $(130 \mu \mathrm{L}$, addition repeated every $24 \mathrm{~h})$ were added and the reaction was stirred at $130^{\circ} \mathrm{C}$ for three days. The mixture was centrifuged and the supernatant was filtered using a $0.1 \mu \mathrm{m}$ PTFE filter (Millipore, USA). The filter was washed 5 times with DMF. The material recovered as functionalised MWNTs $(60 \mathrm{mg})$ onto the filter was then extensively washed with DCM $(100 \mathrm{~mL})$ and dried. $200 \mu \mathrm{L}$ of a DMF solution of $f$-MWNTs (approximately 5 $\mathrm{mg} / \mathrm{mL}$ ) were deposited dropwise with a pipette onto the glass coverslips and the solvent removed by evaporation. The coverslips were kept at $350^{\circ} \mathrm{C}$ under nitrogen atmosphere for $15 \mathrm{~min}$, removed from the oven and sterilized by UV treatment.

\section{Preparation of cultures}

Standard dissociated hippocampal cultures were prepared according to Malgaroli, ${ }^{1}$ with slight modifications. ${ }^{2}$ Hippocampi were dissected from 0 - to 3 -d-old animals killed by decapitation in accordance with regulations of the Italian Animal Welfare Act and approved by the local Authority Veterinary Service. In brief, $400 \mu \mathrm{m}$ thick tissue slices were digested and cells were recovered as described. ${ }^{2}$ Cells were plated on peptide-free glass coverslips and on CNTs treated glass coverslips, coverslips were placed in Petri dishes and cultured in serum-containing medium in a $5 \% \mathrm{CO}_{2}$ humified incubator for 7-9 days.

\section{Electrophysiological recordings}


Cultures were superfused with a solution containing (mM): $\mathrm{NaCl} 156, \mathrm{KCl} 4, \mathrm{MgCl}_{2}$ $1, \mathrm{CaCl}_{2} 2$, HEPES 10, glucose $10 .^{3}$ Recordings were obtained from neurons with patch pipettes (4-7 M $\Omega$ ) using an Axopatch 1-D (Axon Instruments, Foster City, CA, USA). Pipettes were filled with a solution containing (mM): K gluconate 120, KCl 20, HEPES 10, EGTA 10, $\mathrm{MgCl}_{2} 2$ and $\mathrm{Na}_{2}$ ATP 2 (pH 7.35 adding KOH). In voltage clamp, all recordings were taken at the same holding potential of $-58 \mathrm{mV}$. Membrane potentials were corrected for liquid junction potentials. The uncompensated value for series resistance was 8-10 M $\Omega$.

\section{Immunocytochemistry and image analysis}

For SEM analysis, cultures (control and CNTs) after 8-10 days in vitro, were fixed with a solution containing $2.5 \%$ glutaraldehyde in $0.1 \mathrm{M}$ cacodylate buffer, $\mathrm{pH} 7.4$, for 1 hour at RT and then washed in cacodylate buffer, transferred into a PBS solution containing $1 \%$ osmium tetroxide for 1 hour at RT. Cultures were then dehydrated and processed for standard SEM analysis.

For immunocytochemistry cultures (control and CNTs) were fixed with $3 \%$ paraformaldehyde in PBS for $15 \mathrm{~min}$ at room temperature (RT), rinsed 3 times in PBS, incubated in $3 \%$ normal goat serum, $0.3 \%$ Triton X-100 in PBS (blocking solution), for $30 \mathrm{~min}$ at RT and subsequently incubated with the following antibodies: mouse monoclonal anti-microtubule associated protein 2 (MAP-2; Sigma), at 1:400 dilution, mouse monoclonal anti-glial fibrillary acidic protein (GFAP; Sigma), at 1:200 dilution. The incubation was for $16 \mathrm{hr}$, at $4^{\circ} \mathrm{C}$ in a humid chamber. Cultures were then processed with the corresponding biotinylated secondaries as previously described. ${ }^{4}$ Slides were viewed with a Zeiss Axioskop (Germany) microscope equipped with an Optronics Magnafire CCD camera (Optronics, Italy). The morphological data were obtained using the Image pro express program (Media 
Cybernetics, Des Moines, IA). We observed 20 different coverslips in every growth condition and not less than 50 cells or 10 different fields per coverslips were analyzed. Between 200 and 250 cells per condition were selected at random for analysis and a digital image of the MAP-2 positive cell was captured. We quantified the number of positive-cells per unit area, the area of the cell body, the number of processes exiting each cell. For these two last measurements only clearly distinguishable isolated neurons were chosen. A neuronal process was defined as a tapering, MAP-2-stained process that was greater than $20 \mu \mathrm{m}$ in length. From the GFAP-positive glial cells we determined the morphology and the number of astrocytes per unit area in the two growing conditions.

\section{Data acquisition and analysis}

Signals were filtered at $10 \mathrm{kHz}$, digitally recorded on a computer using the CLAMPEX 8.1 software (Axon Instruments) and stored for later analysis. In voltage clamp recordings, PSCs were analyzed as described elsewhere ${ }^{3}$; at least $200 \mathrm{~s}$ of each recording were analyzed, frequency and amplitude of events were determined as described in details elsewhere. ${ }^{3}$ In a group of experiments spontaneous excitatory PSCs were recorded at a holding potential of $-40 \mathrm{mV}$, to avoid contamination with $\mathrm{GABA}_{\mathrm{A}}$-receptor mediated inhibitory currents ${ }^{5}$, since under our experimental conditions this value is similar to the reversal potential for chloride ions ${ }^{6}$. Membrane capacitance and input resistance were measured under voltage clamp and were on average $55 \pm 2.8 \mathrm{pF}$ and $244 \pm 25 \mathrm{M} \Omega$ in control neurons ( $\mathrm{n}=15$ cultures), displaying substantially similar values to those of neurons grown on CNTs $(58 \pm 3.3 \mathrm{pF}$ and $265 \pm 24 \mathrm{M} \Omega$, for capacitance and resistance, respectively, $n=15$ cultures). Intrinsic excitability was measured in current clamp mode. Resting membrane potential was similar under the two culturing conditions, with values on average of $-52.6 \pm 1.4$ and of 
$-53.7 \pm 1.2 \mathrm{mV}$, in control and CNTs respectively. All values were in good agreement with previous studies on cultured hippocampal neurons ${ }^{7}$. Action potentials were detected and analyzed with the pClamp 9 program (Axon Instruments) and action potential heights and halfwidths were measured off-line. Numerical data in the text are expressed as means \pm s.e.m. with $n=$ number of cultures, unless stated otherwise. For statistical comparison data from CNTs cells were tested against data obtained from a general pool of control, aged-matched cultures or against control cells from the same culture series (named sister cultures). Statistical analysis was carried out using $t$ test or ANOVA and considered significant at the $p<0.05$ level, unless stated otherwise.

\section{References}

1. Malgaroli, A. and Tsien, R.W. Nature 357, 134-139 (1992).

2. Tongiorgi, E., Righi, M. and Cattaneo, A. J. Neurosci. 17, 9492-9505 (1997).

3. Galante, M., Nistri, A. and Ballerini, L. J. Physiol. 523, 639-651 (2000).

4. Avossa, D., Rosato-Siri, M.D., Mazzarol, F., and Ballerini, L. Neuroscience 122, 391-405 (2003).

5. Andjus, P.R., Stevic-Marinkovic, Z. and Cherubini, E. J. Physiol. 504, 103-112 (1997).

6. Ballerini, L., Galante, M., Grandolfo, M., Nistri, A. J. Physiol. 517, 459-475 (1999).

7. Mangan, P.S. and Kapur, J. J. Neurophysiol. 91, 946-957.(2004). 